\title{
Grenzen und Migration - eine dynamische Interdependenz
}

\author{
Bastian A. Vollmer und Franck Düvell
}

\begin{abstract}
Zwischen Grenzen und Migration besteht eine Wechselbeziehung voller Ambivalenzen und humanitärer Implikationen. Definitorisch sind sowohl politisch-territoriale Grenzen wie auch internationale Migration politische Konstruktionen des Staates und seiner Akteure. Ein kurzer Überblick dieses Feldes zeigt dessen Komplexität und die dementsprechend große Diversität an Forschungsansätzen und eine zunehmende Pluralität des Gegenstands Grenze im Feld der Migration. Die Figur und Perspektive der Migranten und Migrantinnen wird bei diesem Überblick in den Vordergrund gestellt. Forschungsdesiderata sind einerseits die vernachlässigte empirische Erforschung der tatsächlichen Abläufe an Grenzen sowie andererseits die Berücksichtigung einer Perspektive in der Diskursforschung.
\end{abstract}

\section{Schlagwörter}

Migration, Grenzen, (Un-)Sicherheit, Staatlichkeit, Politik

\section{Einleitung}

Grenzen im Feld der Migration ${ }^{1}$ waren und sind mit steigender Signifikanz eine politische Konstruktion. Im vorliegenden Beitrag wird der Zusammenhang von Migration und Grenzen unter besonderer Berücksichtigung der politischen Dimension dargestellt. Der Artikel wird in einem ersten Teil dazu einen kurzen Überblick dieses Feldes bieten, wobei die Komplexität und expandierende Diversität an Forschungsansätzen und in einem zweiten Teil die Pluralität des Gegenstands Grenze im Feld der Migration gezeigt wird. Es wird in diesem Beitrag die Figur und Perspektive der Migranten und Migrantinnen in den Vordergrund gestellt und Forschungsdesiderata der empirischen Erforschung der tatsächlichen Abläufe an Grenzen benannt. Ein letzter Teil wird anschließend Perspektiven der Diskursforschung vorstellen und weiterführende Fragestellungen vorschlagen.

In der Vormoderne wurden Grenzen durch natürliche Barrieren wie Flüsse, Meere oder Gebirgszüge sowie landschaftliche Markierungen wie Gräben, Bäume oder Feldreihen, aber auch durch menschgemachte Frontlinien zwischen zwei Armeen sowie architektonische Konstruktionen wie den Limes oder die Chinesische Mauer repräsentiert (vgl. Waldron 1990; Heather 2010). Demgegenüber haben Abgrenzungen im Zeitalter der Emergenz von Nationalstaaten, Staatlichkeit, Staatsbürgertum und territorialen Ansprüchen eine andere Qualität und Bedeutung erhalten (vgl. Herrmann/Vasilache in diesem Band). Gleichzeitig wanderten und veränderten sich Grenzen seit der Erfindung und Entwicklung des Staates (vgl. Anderson 1997). Staaten gingen unter, Staatsterritorien wurden verkleinert oder erweitert und mit ihnen verschwanden Grenzen oder wurden neue geschaffen. Manche Menschen, wie zum Beispiel in der heutigen Westukraine, lebten, ohne ihren Lebensstandort zu verlassen, in mehreren verschiedenen Staaten.

Im Jahr 2017 wurden weltweit 1,2 Milliarden internationale Einreisen mit zumindest einer Übernachtung verzeichnet, dies ist nahezu eine Verdoppelung seit 1997 (vgl. UNWTO 2016,

1 Migrantische Bewegungen inkludieren Fluchtbewegungen. 
2018). Im selben Jahr wurden 258 Millionen internationale Migrantinnen und Migranten registriert, eine Zunahme von 120 Millionen seit 1990, der Anteil von Migrantinnen und Migranten an der Weltbevölkerung ist seit 1990 um 50 Prozent auf nunmehr 3,4 Prozent angestiegen (vgl. UN 1997; UNDESA 2018). Darin enthalten sind 25,4 Millionen Flüchtlinge, die in einem anderen Staat Schutz suchen, was ebenfalls nahezu eine Verdoppelung seit 1997 darstellt (vgl. UNHCR 1997, 2018). Hinzu kommt eine unbekannte Zahl von irregulären Migrantinnen und Migranten, in den ersten Jahren des 21. Jahrhunderts dürfte es sich um 20 bis 40 Millionen weltweit gehandelt haben (vgl. Düvell 2006) - dies hat sich seither nicht fundamental geändert. Während die absoluten Zahlen demnach seit Ende der 1990er-Jahre stark angestiegen sind, bleiben sie aber proportional zur ebenfalls wachsenden Weltbevölkerung etwas zurück, zudem zeigt sich der Anteil von Migrantinnen und Migranten sowie Flüchtlingen an internationalen Reisen wie auch an der Weltbevölkerung sehr gering und damit eher als Ausnahmeerscheinung. Allerdings geben weltweit weitere 710 Millionen Menschen an, immerhin 10 Prozent der Weltbevölkerung, migrieren zu wollen (vgl. Gallup 2017).

\section{Migration als Gegenstand der Forschung}

Nationalstaaten üben Souveränität über ihr Territorium aus und entwickeln Migrationspolitiken, Institutionen und Gesetze sowie Praktiken und Diskurse (vgl. Betts 2011), um das Verhalten von Menschen im Hinblick auf Auswanderung, Einwanderung, Rückkehr und Integration vor dem Hintergrund nationaler wirtschaftlicher, demografischer und politischer Interessen zu steuern (vgl. United Nations 2013). Demnach bestimmen sie über die Aufnahme und deren Bedingungen oder auch Abweisung von Bürgern und Bürgerinnen anderer Staaten. Dies spiegelt sich u.a. in der Visapolitik oder der Tatsache wider, ob z.B. die Einreise ohne Visum möglich ist oder ein Visum und damit eine Kontrolle erst bei oder bereits vor Einreise erforderlich ist. Die Gesamtheit dieser Politiken, Praktiken und damit zusammenhängenden Diskurse kann auch als Migrationsregime bezeichnet werden (vgl. Collinson 1993; siehe auch Krasner 1982).

Grenzen zwischen Nationalstaaten sind dabei nur ein Element von Migrationspolitik (vgl. Vogel 2003), spielen aber eine entscheidende Rolle bei der Definition, Registrierung und Kontrolle von Reisenden, Migrierenden und Flüchtenden. Für die meisten Reisenden ist eine Grenze kaum mehr als eine Institution, an der man, wenn überhaupt, auf Aufforderung von Grenzpolizist*innen bei der Aus- und/oder Einreise die Identitätsdokumente vorweist, wodurch die Reise kurz aufgehalten wird. Ärgerlich wird es vor allem dann, wenn dies zu längeren Wartezeiten führt oder man sogar zurückgehalten, abgewiesen und zurückgeschickt wird. Im Sommer 2015 konnte man in Europa nicht nur die Grenzübertritte von über einer Millionen Menschen beobachten, die keine Einreiseerlaubnis hatten, es kam sogar zu gewalttätigen Zusammenstößen zwischen einer besonderen Kategorie von Reisenden, Migrantinnen und Migranten, oder genauer gesagt Flüchtlingen, die Einlass forderten, und Grenzschutztruppen, insbesondere in Mazedonien und Ungarn, die dies zu verhindern suchten (vgl. Düvell 2017). In diesem Zusammenhang macht die Menschenrechtsorganisation United for Intercultural Action (2018) darauf aufmerksam, dass entlang der Außengrenzen der Europäischen Union regelmäßig Menschen bei dem Versuch, ohne die benötigten Papiere und Erlaubnisse einzureisen, ihr Leben riskieren oder gar zu Tode kommen: 34.361 Opfer wurden zwischen 2009 und 2018 bekannt.

Internationale Migration ist ein gesellschaftliches und politisches Konstrukt. Es ist die politische Organisation der Menschheit in Nationalstaaten, die Definition von geografischem Raum 
als souveränem Raum, die Zuteilung von gesellschaftlicher Zugehörigkeit nach dem Prinzip der Nationalität und die daran gekoppelte Zuteilung von Ressourcen, Rechten und Mitgliedschaftsregimen, die räumliche Mobilität als internationale Migration konstruiert (vgl. auch Schindler in diesem Band). Dies wird unter anderem an dem Gegenbeweis deutlich, wonach für die geografische Mobilität innerhalb eines Nationalstaates in der Regel die Freizügigkeit gilt und sie dementsprechend in der Regel nicht als Migration im politischen Sinne verstanden wird, ebenso wenig spricht man bei Ortswechseln innerhalb der Europäischen Union - wo Grenzkontrollen abgeschafft wurden - politisch von Migration, sondern oft vielmehr von Mobilität (vgl. Europäische Kommission 2018). Dies zeigt, dass Mobilität innerhalb von Staaten überwiegend als selbstverständlich hingenommen, sie aber zwischen Staaten als normatives Problem betrachtet wird (vgl. Bommes/Geddes 2000). Daneben spielt Temporalität bei der Konzeptualisierung von menschlicher geografischer Bewegung eine Rolle. Die Vereinten Nationen, vor allem die Abteilung für Bevölkerung (vgl. UNDESA 1998), definieren temporäre Migration als die Anwesenheit in einem anderen Nationalstaat von mehr als drei aber weniger als 12 Monaten und sprechen von Immigration, als einem dauerhaften Phänomen, wenn der Aufenthalt 12 Monate oder länger dauert. Demgegenüber definiert die UN-Unterorganisation International Labour Office (2015) jeden Aufenthalt in einem anderen Staat zum Zweck der Arbeit als Arbeitsmigration, ganz unabhängig von der Aufenthaltsdauer. Zudem werden nur zwangsweise Vertriebene, die eine staatliche Grenze übertreten, als Flüchtlinge betrachtet, alle anderen gelten als intern Vertriebene. Allerdings gelten international Vertriebene ebenfalls vom ersten Tag an als Flüchtlinge und das Kriterium der Dauer fällt weg (vgl. UNHCR 2020). Mitunter weichen auch nationale Praktiken von denen der UN ab. Diese zeigt, dass das Kriterium Zeit sowohl willkürlich festgelegt wird als auch uneinheitlich geregelt ist (vgl. Leutloff-Grandits in diesem Band).

Grenzen oder präziser die Außengrenzen von Nationalstaaten spielen demnach bei der Konstruktion von Migration und Flucht eine zentrale Rolle. Zum einen wird geografische Mobilität erst durch die Übertretung einer solchen Grenze als Flucht oder Migration definiert. Zum anderen ist es die Grenze beziehungsweise sind es die Bürokratien und Institutionen, die an dieser imaginären Linie zwischen zwei souveränen Territorien eingerichtet und eingesetzt werden, die Migration als solche registrieren, kontrollieren und entweder zulassen oder verhindern. Grenzen und die dort in Stellung gebrachten Behörden sind zudem ein wichtiges Mittel der Durchsetzung von Migrations- und Grenzpolitik. An der Grenze werden Zäune, Wachtürme, Minenfelder, Patrouillen und Kontrollposten installiert, teils werden diese auch ins Hinterland ausgedehnt, aber immer im Hinblick auf die vor- oder nachgelagerte Kontrolle von Bewegung über die eigentliche Grenzlinie hinweg. Dies führt dazu, dass durch Grenzen zusätzliche Migrationskosten entstehen, etwa weil Visa benötigt werden, und dadurch der Preis für Migration insgesamt steigt. Mitunter erhöhen Grenzen auch das Risiko von Migration, etwa wenn die Migrierenden keine Erlaubnis erhalten und dementsprechend klandestine Formen und Routen wählen (vgl. Eschbach et al. 1999).

Bislang wenig erforscht ist eine bedeutende Konsequenz von Grenzen: die Durchsetzung von Immobilität oder Sesshaftigkeit (vgl. dazu auch Nail in diesem Band). Inwiefern besteht also die Auswirkung von Grenzen darin, Migration zu verhindern? Nichtereignisse wie etwa Nichtmigration wecken selten das Forschungsinteresse. Ausnahmen sind hier die Untersuchungen von Flüchtlingen und Migranten und Migrantinnen in Staaten entlang der Außengrenzen der EU, wie etwa in der Türkei, Libyen und Ukraine, die dort gestrandet sind (vgl. Düvell 
et al. 2014). Sie beschäftigen sich mit den problematischen und oft sogar menschenrechtsverletzenden Lebens- oder Haftbedingungen jener, denen die Einreise verunmöglicht oder verweigert wurde. Bestimmte Orte und Räume, wie der Fluss Evros zwischen der Türkei und Griechenland, die Ägäis-Insel Lesbos sowie Lampedusa und die Straße von Sizilien haben in diesem Zusammenhang notorische Berühmtheit erlangt. Schließlich geht Carling (2002; siehe auch Houtum/Naerssen 2001) soweit, die gegenwärtige Debatte umzudrehen und spricht von einem Zeitalter der unfreiwilligen Immobilität statt von einem Zeitalter der Migration (vgl. Castles/Miller 1998). Dies ist angesichts des kleinen Anteils weltweiter Migranten und Migrantinnen eine durchaus plausible Lesart.

An der internationalen Grenze stoßen deshalb die Ziele eines Staates - beziehungsweise der Bürger dieses Staates - und die Aspirationen von Angehörigen anderer Staaten und damit von Individuen aufeinander (vgl. Shrestha 1987). Grenzen sind demnach nicht nur die Faltlinien zwischen zwei politischen Mächten, sondern determinieren Inklusions- und Exklusionsprozesse und können sogar „Faltlinien des Überlebens“ darstellen (vgl. Petryna/Follis 2015). Insofern sind sie ein Ort des Zusammentreffens zwischen der Macht des Staates und menschlicher Wirkungsmacht (buman agency) und damit letzten Endes der Ort einer potenziellen sozialen Konfrontation zwischen potenziell entgegengesetzten Zielen und Ansprüchen (vgl. z.B. Rodriguez 1996). Migration, so Glick-Schiller et al. (1995, S. 50) „ist ein wichtiges Mittel, durch das Grenzen angefochten und übertreten werden“. Zwar verhindert eine Vielzahl von Faktoren (z.B. Mangel an finanziellen Mitteln, sozialen Netzwerken, Humankapital) die Realisierung der Migration jener 710 Millionen, die eine dementsprechende Aspiration haben, aber Grenzen und dort durchgesetzte Aus- und Einreisebeschränkungen spielen ebenfalls eine bedeutende Rolle. Allerdings verläuft der Großteil der internationalen Mobilität und Migration innerhalb der Gesetze, das heißt, die Ziele von Staaten und die Aspirationen von Individuen sind entweder teils deckungsgleich oder führen in der Regel zumindest nicht zu einem offenen Zusammenstoß. Dennoch sollte das Konfliktpotenzial nicht überschätzt werden.

An der Grenze manifestiert sich auch der Unterschied zwischen erlaubter und unerlaubter Migration, der Gegensatz von Inklusion und Exklusion, der Kampf von Ordnung und Unordnung, der (potenzielle) Widerspruch zwischen politischen Zielen und individuellen Aspirationen sowie zwischen Staat und Individuum. Irreguläre Migration, unerlaubte Grenzübertritte, Proteste gegen geschlossene Grenzen sowie die Konsequenzen nicht zugelassener Migration sind deshalb auch die deutlichste Manifestierung der normativen Probleme mit Grenzen.

\section{Zentrale Konzepte und Grundannahmen der Migrations- und Migrationspolitikforschung}

Die Grenzforschung innerhalb der Migrations- und Migrationspolitikforschung hebt sich von der allgemeinen Grenzforschung dadurch ab, dass sie sich vor allem mit dem Wechselverhältnis von Grenze und menschlicher Mobilität befasst, und zwar sowohl im Hinblick auf die eigentliche Wanderung als auch im Hinblick auf die nachgeordneten In- und Exklusionsprozesse. Für einige Autorinnen und Autoren ist Migration nur mehr ein Prisma, um andere Themen wie die Transformation von Territorialität, Souveränität und politischer Imagination zu untersuchen (vgl. Walters 2009), für andere ist Migration der zentrale Forschungsgegenstand, in dem Grenze nur implizit auftaucht (vgl. z.B. Triandafyllidou/Maroukis 2012; Crawley et al. 2017). 
Die Forschung zu Migration und Grenze befasst sich einerseits mit den Erfahrungen von Migrierenden mit Grenze oder mit der Um- und Durchsetzung von Grenze und wird von einigen wenigen Themen dominiert:

a) irreguläre, also unerlaubte Migration und Grenzübertritte (vgl. z.B. Düvell 2006) sowie die damit zusammenhängende sogenannte Transitmigration (Collyer et al. 2012). Insbesondere erhält die Migration mit Booten von sogenannten boat people oder boat refugees aufgrund ihrer Sichtbarkeit und Devianz besondere Aufmerksamkeit (vgl. Mountz 2010; Weber/Pickering 2011; Crawley et al. 2017);

b) die Vorverlagerung von Migrationskontrollen in Nachbarstaaten, insbesondere die der Europäischen Union, die als Externalisierung oder Extraterritorialisierung von Migrationskontrolle bezeichnet wird (vgl. z.B. Ryan/Mitselegas 2010; Gammeltoft-Hansen 2011);

c) die Wirksamkeit, Digitalisierung (vgl. Broeders 2007) und Militarisierung von Migrationskontrolle (vgl. Lutterbeck 2006) sowie die Rolle von Sicherheitsüberlegungen und -diskursen bei der Gestaltung von Migrationspolitik, die als Versicherheitlichung von Migration analysiert wird (vgl. Adamson 2006; Vollmer 2014);

d) die Ein- und Ausgrenzungsfunktion von Grenz- und Migrationspolitik innerhalb von Staaten (vgl. z.B. Takikawa 2016);

e) die Privatisierung und Kommerzialisierung von Migrations- und Grenzkontrolle (vgl. Infantino 2016);

f) die humanen Kosten von Ausgrenzung und damit zusammenhängend Fragen von Verantwortung (vgl. Weber/Pickering 2011) und Ethik (vgl. Pecoud/Guchtenaire 2006; Bauder 2017) sowie

g) transnationale Praktiken (transnationale Migration, Diasporas; vgl. Basch et al. 1994), die die Idee des Nationalen und damit die abgrenzende Funktion von Grenze unterminieren und individuelles deviantes Verhalten, welches u.a. als Widerstand oder Autonomie der Migration diskutiert wird (vgl. Moulier-Boutang 2002), aufzeigen.

Bei all diesem stehen hauptsächlich die Außengrenzen der EU und der USA im Fokus; andere Grenzen, z.B. die der Staaten im Mittleren Osten, in Südasien oder Zentralasien, finden vergleichsweise wenig Beachtung (Ausnahmen u.a. bei Raghuram/Piper 2011).

Die Forschung zu Grenze und Migration nimmt je nach Disziplin spezifische Perspektiven ein, die hier einmal zugespitzt und vereinfacht zusammengefasst werden. Ethnologinnen und Ethnologen und teils auch Humangeografinnen und Humangeografen geht es, vereinfacht ausgedrückt, eher um die Mikroebene und die Verhaltensweisen sowie Deutungsprozesse der Migrierenden sowie um die Interaktionen der beteiligten Akteure und Akteurinnen: der Migrierenden, deren Helfern und Helferinnen (,Schmugglern') sowie den street level bureaucrats, welche gemeinsam die „Grenze täglich herstellen“ (Mountz 2010, S. xix). Historikerinnen und Historiker weisen beispielsweise nach, dass Migration ein anthropologisches Kontinuum ist (vgl. Hoerder 2002), während Grenzen und Migrationskontrollen eher neuzeitliche Phänomene darstellen (vgl. Caplan/Torpey 2001). Politikwissenschaftlerinnen und Politikwissenschaftlern hingegen geht es um die Rolle von Staat und Politik und insbesondere um die politische Beeinflussung von menschlichem (Migrations-)Verhalten oder auch dessen Scheitern, wobei sie auch Diskurse analysieren (s.u.). Soziologinnen und Soziologen analysieren Gesellschaft, gesellschaftliche Organisation und Zusammenleben, Ungleichheit, Ausgrenzung und in diesem Kontext auch die gesellschaftliche Funktion von Grenzen, etwa die Konstruktion ungleicher Rechte sowie um Rassismus (vgl. Faist 2016). Juristinnen und Juristen studieren nationales 
und internationales Flüchtlings- und Migrationsrecht sowie die teilweise bestehenden Widersprüche zwischen diesen und damit auch die normative Seite der Funktion von Grenze. Kriminologen und Kriminologinnen untersuchen die strukturelle Gewalt und Verantwortung für die Opfer von Grenzkontrollen (vgl. Weber/Pickering 2011). Und in den Wirtschaftswissenschaften werden der Nutzen von Orten für Migrierende, rationale Wahlhandlungen, der Einfluss von Migration auf Arbeitsmarkt, Einkommen und Steueraufkommen sowie die Rolle von Grenze bei der Determinierung der Rahmenbedingungen untersucht (vgl. Goldin 2018).

In einem Feld wie der Migrationsforschung werden diese disziplinären Grenzen allerdings häufig überwunden, wobei die Übergänge fließend sind. Daraus resultiert eine ganze Bandbreite von dichten und teils separaten Diskursen und Forschungstraditionen, wovon im Folgenden nur die wichtigsten Perspektiven vorgestellt werden. Aus Platzgründen kann nicht auf alle Positionen im Einzelnen eingegangen werden.

Innerhalb dieser diversen Studien verfolgt eine prominente Gruppe von Wissenschaftlerinnen und Wissenschaftlern einen eher philosophischen Foucault'schen Ansatz, sowohl in der Analyse der Institution Grenze als auch von individuellem Verhalten. Grenzpolitik wird hier auch als Biopolitik verstanden, d.h. als Politik und Ausübung von Macht, die ein Bevölkerungsmanagement zum Ziel hat (vgl. z.B. Walters 2002). Ein weiterer, teils verwandter, eher systemischer und teils normativer Forschungsstrang interpretiert Grenze (border) als Abgrenzung (bordering) und formuliert die These "Grenzen sind überall“ (vgl. z.B. Balibar 2004 oder auch Rumford 2006). Um bei dieser Vielfalt von Grenz- und Abgrenzungsprozessen die analytische Schärfe nicht zu verlieren, sollte aus unserer Sicht zwischen vorgelagerten (Visa-)Kontrollen, Grenz- und Migrationskontrollen an den äußeren Staatsgrenzen sowie Kontrollen an den internen administrativen Grenzen und den diese Kontrollen durchführenden unterschiedlichen Agenturen (Botschaft, Grenzschutz, Polizei und zivile Behörden) unterschieden werden.

Eine weitere prominente Forschungslinie übt fundamentale Kritik an der Kontrolle und Beschränkung von Migration. Etliche Autoren und Autorinnen (vgl. u.a. Hayter 2000; Pecoud/Guchtenaire 2006) stellen fest, dass es im internationalen Recht der Erklärung der Menschenrechte, Artikel 13, zwar ein Recht auf Verlassen eines Staates, aber kein Recht auf Einreise in einen anderen gibt. Diese Inkonsistenz wird zum Anlass genommen, für offene oder keine Grenzen sowie ein Recht auf Migration einzutreten. Andere Autorinnen und Autoren hinterfragen den Nutzen von Grenzen, zeigen, dass Grenzbarrieren und Grenzen deshalb teilweise wirkungslos seien, und implizieren demnach, dass Grenzen bzw. Grenzkontrollen in der bisherigen Form abgeschafft gehören. So weist Jagdish Bhagwati (2003) darauf hin, dass irreguläre Migration trotz intensivierter Kontrollen sogar noch weiter zunimmt, Franck Düvell (2006) zeigt, dass die Anziehungskraft von Arbeitskraftnachfrage, aber auch menschliche Wirkungsmacht stärker sind als Migrations- und Grenzkontrollen. Und der Ökonom Nigel Harris (2007) hält Grenzen für ökonomisch unsinnig, da sie die freie Migration von Arbeit nach den Gesetzen von Angebot und Nachfrage blockieren und für diverse ökonomische und politische Verzerrungen sorgen.

Ein anderer Forschungsstrang geht mikrosoziologisch und ethnografisch vor. So untersuchen Federica Infantíno (2016) sowie Vassilis Tsianos und Sabine Hess (2010) Migrationskontrollen bzw. die Implementierung von Visapolitik außerhalb der Grenzen der Europäischen Union. Martin van der Velde und Ton van Naerssen (2015) untersuchen Grenze aus der Perspektive der unterschiedlichen menschlichen Perzeptionen sowie Reaktionen und Entscheidungsfindungsprozesse und damit das Zusammenspiel von strukturellen und kognitiven Faktoren. Sie 
zeigen einerseits auf, dass Grenzen als Schwelle von Migration fungieren, aber auch, dass Grenzen nicht absolut sind, sondern dynamisch und abhängig von den sich verändernden Bedingungen auf beiden Seiten der Grenze. Zudem wirken sie sich auf unterschiedliche Individuen verschieden aus. Schließlich verfasste Shahram Khosravi (2010) eine vielbeachtete Autoethnografie eines „illegalen Reisenden“ und dessen Formen des Widerstands. Er analysiert Grenze eher aus der Perspektive des Individuums, welches auf die Macht, die an der Grenze ausgeübt wird, mit Widerstand reagiert (vgl. auch Rodriguez 1996; Yaghmaian 2005).

Demnach befassen sich Grenzstudien aus ethnografischer oder soziologischer Perspektive in methodischer Hinsicht eher mit Phänomenen von unten und untersuchen z.B. die Praktiken Migrierender und Flüchtender. Aus einer eher politikwissenschaftlichen, kriminologischen oder normativen Perspektive von oben wird vornehmlich Grenze als Institution erforscht. Eine dritte minoritäre Zwischenperspektive untersucht zwar auch die Grenze, aber nicht politikwissenschaftlich, sondern ebenfalls ethnografisch aus der Perspektive der individuellen Akteure und Akteurinnen (street level bureaucrats, vgl. u.a. Tuckett 2018).

Nahezu alle Studien im Feld Grenze und Migration sind von qualitativer, normativer oder philosophisch-theoretischer Natur, einige wenige gehen gar mit forensischen Methoden an das Feld heran (vgl. Weber/Pickering 2011; Last et al. 2017). Quantitative Erhebungen zur Durchsetzung von Grenze oder zur Erfahrung von Grenzübertritten sind selten, wenn nicht gänzlich absent. Deshalb lässt sich bislang auch wenig statistisch Belastbares über die Wirkung von Grenze sagen. Beispielsweise kann nicht bestimmt werden, ob und welchen Abschreckungseffekt Grenze, Grenzkontrollen und die Risiken eines unerlaubten Grenzübertritts auf die 710 Millionen Migrationswilligen (vgl. Gallup 2017) weltweit haben.

\section{Perspektivische Weiterentwicklungen}

Strömungen der Grenzforschung im Bereich Migration werden immer deutlicher von alternativen und innovativen Perspektiven aus inter- und transdisziplinären Feldern u.a. der Anthropologie, Geografie, Soziologie sowie der Rechtwissenschaft und der Politikwissenschaft erweitert. Ende der 1980er-Jahre wurden wissenschaftliche Sichtweisen der Grenzforschung durch neue und drastische Bewegungen von Grenzen (territorial und politisch) liberalisiert. In der Entwicklung im Feld Grenzen und Migration wurde zunehmend die vorherrschende Perspektive einer Analyse von innen nach außen hinterfragt (vgl. Walker 1993). Einen Perspektivwechsel vorzunehmen und eine andere Wahrnehmung der äußeren Seite der Grenze aufzunehmen oder die Grenze als eigenständigen Forschungsgegenstand zu sehen, wurde über viele Jahre vernachlässigt, ist inzwischen aber ein Kernbestandteil vieler migrantischer Bewegungen und auch einer steigenden Zahl von Studien geworden (s.o.). Begriffe wie Bedrohung, Kontrolle, Überwachung und Undurchlässigkeit wurden entsprechend neu diskutiert (vgl. Bigo 2002; Huysmans 2006; Balibar 2009; Vaughan-Williams 2009; Bauman/Lyon 2012; Fassin 2012). Zunehmend wurden Grenzen jedoch als Barrieren zur Disposition gestellt und im Fall von Staatsgrenzen die Manifestation dieser staatspolitisch konstruierten Barriere und ihrer verschiedenartigster Repräsentation infrage gestellt (vgl. Genova 2002; Shields 2006; Vollmer 2012; Papadopoulos/Tsianos 2013; Cooper/Rumford 2013).

Behält man aber die Perspektive der sich bewegenden Menschen im Auge - wie oben als mikrosoziologischer/ethnografischer Forschungsstrang aufgeführt -, dann erscheinen Grenzen als Gefährdung und lebensbedrohliche Realitäten für migrantische Bewegungen. Damit drän- 
gen sich neue und andere Forschungsansätze in den Vordergrund und ermöglichen neue Analysen zum Gegenstand Grenze. Die Perspektive der Migration und die der Migranten und Migrantinnen trägt zum Verständnis und zu einer Neukonfiguration von Grenzen bei, d.h. dieser Ansatz dreht die Perspektive auf Grenzen um. Grenzen sind demnach nicht nur schlicht Anlagen, Institutionen oder Symbole sowie Instrumente zur Durchsetzung von Sicherheit, sondern Ursache von Unsicherheit. Dadurch ändert sich das Verständnis von Grenzen und ihren primären Funktionen (vgl. Schwell in diesem Band).

Im Prozess einer migrantischen Bewegung finden multiple Entscheidungsfindungen statt, die auf einer Genese von Imaginationen und Aspirationen basiert (vgl. Carling 2002). Imaginationen anderer Ort und Räume werden produziert und mit Aspirationen verknüpft. Hoffnung kann als eine der zentralen Kräfte einer Entscheidungsfindung der Migration bezeichnet werden (vgl. Appadurai 2013). Zu differenzieren sind diese Prozesse im Fall einer erzwungenen Bewegung. Der Verlauf dieser Prozesse findet in einer anderen Zeitspanne und unter einer erhöhten Situation von Gefahr bis hin zu Ausweglosigkeit und Todesängsten statt. Staatliche Grenzziehungen verschiedenartiger Form stellen im Prozess der Migration zusätzliche Hindernisse dar, die die Bewegungen von Menschen erschweren, aufhalten oder auch beenden können. Dadurch können migrantische Imaginationen und Aspirationen zu Illusionen und Enttäuschungen werden. Gleichzeitig zeugen Migrationserfahrungen und Verläufe von einer hohen Diversität und erzeugen verschiedenste Narrative. Beispielsweise konnte Bastian Vollmer (2016) zeigen, dass die EU-Außengrenze von Ukrainern und Ukrainerinnen, die eine Emigration in Erwägung ziehen, als Problem, Barriere oder auch als abstoßend, ausgrenzend und desillusionierend erfahren wird. Andererseits können Grenzen auch genutzt werden und haben in manchen Fällen sogar eine verbindende Wirkung. Das Phänomen findet sich an Grenzgebieten weltweit (vgl. u.a. Paasi/Prokkola 2008). Chris Rumford und Anthony Cooper (2013) gehen einen Schritt weiter und sehen das Potenzial, dass Grenzen eine effektive Konnektivität erzeugen können, d.h. nicht Teilung, sondern Konnektivität durch erweiterte Netzwerke repräsentieren können.

Der empirische Ansatz aus einer migrantischen Perspektive (vgl. z.B. Khosravi 2010) eröffnet aber alternative Narrative der Grenze über Harmlosigkeit, Überflüssigkeit oder einen bump on the road, um den es sich lediglich zu kümmern gilt, der ein Management der Umstände benötigt, wie es Vollmer (2012) in seinem Beitrag beschreibt. Damit verkörpert Grenze, in diesem Fall die EU-Außengrenze, nicht nur eine Gefahr oder eine festungsartige Abschreckung, sondern gegebenenfalls nur ein kurzzeitiges Hindernis, das zu überschreiten ist und beinahe einer Spielerei gleicht. Diese Perspektive weist auf die Autonomie von Migranten und Migrantinnen hin. Es zeigt die Wirkungsmacht der sich bewegenden Menschen gegenüber dem scheinbaren Bollwerk Grenze. Die Figur des Migranten bzw. der Migrantin repräsentiert einen wirkungsmächtigen und autonomen Akteur und wird nicht zu einem verletzbaren Objekt reduziert (vgl. u.a. Papadopoulos/Tsianos 2013). Allerdings soll auch keine Romantisierung von Migration mit dem Begriff der Autonomie einhergehen (vgl. Mezzadra 2010; Scheel 2013). Vielmehr werden durch diesen Blickwinkel die Prozesse der Entrechtung, der Exklusion und des „humanitären Grenzspektakels“ (vgl. Cuttitta 2014, S. 200) aus einer ,direkten“ und nichtmedialisierten Quelle beschrieben. Das Eigene der migrantischen Subjekte steht hier im Zentrum des Ansatzes. Migrantische Subjektivität ist jedoch nicht lediglich als empirische Datenquelle zu sehen, sondern diese „Praktiken der Migration“ (Hess/Tsianos 2010, S. 44; Hess/Schmidt-Sembdner in diesem Band) sind in einem Regime von Grenzen zu situieren und 
zu analysieren. Mit verschiedenen Ansätzen lassen sich durch diese Prozesse und Praktiken der Migration die Funktionen und Konfigurationen von Grenzen erörtern. Dies steht auch im Gegensatz zur Frequenz und Qualität von Medienberichten, die auf die Wahrnehmung von Migration und auf Diskurselemente der Migration im Zusammenhang mit Grenzen zunehmend einwirken. ${ }^{2}$

Die Sicht auf und die Analyse von Diskursen eröffnet eine weitere Perspektive (vgl. Schindel 2016; Lehner/Rheindorf 2018; Rheindorf/Wodak 2020). Die Wahrnehmung und das Verständnis von Migration sind durch sprachliche Konstruktionen und Narrative, die einen Diskurs entstehen lassen können und damit ein bestimmtes Verständnis von Migration schaffen und etablieren, änderbar. Diese sich ständig verändernden Migrationsdiskurse haben damit eine Auswirkung auf die Deutung und Bedeutung von Grenzen.

Vollmer (2017a; 2019) zeigte Verschiebungen in Migrationsdiskursen und Änderungen der Wahrnehmung von Grenzen. Die Bedeutung und der Stellenwert von Grenzen schwanken, jedoch ist im vergangenen Jahrzehnt, insbesondere in den Jahren 2014 bis 2018, ein neuer Stellenwert und ein neues Image von Grenzen im öffentlichen Raum, z.B. der EU, entstanden. Die Signifikanz der Wahrnehmung von Grenzen und ihrer Bedeutung im digitalen, vernetzten Zeitalter verzeichnet einen expansiven Trend durch eine symbolische Verunsicherung und imaginiert „innere Bedrohung“ (vgl. z.B. Buzan 1991; Côté-Boucher et al. 2014). Jedoch hat sich nicht nur die diskursive Bedeutung und die Symbolik der Grenze erweitert, sondern auch die Praxis der Staatsgrenze. Kontrollmechanismen sind nicht lediglich linear, also an der territorialen Grenzziehung an sich, zu finden, sondern an unzähligen weiteren Kontrollpunkten innerhalb des Territoriums, wie z.B. Bahnhöfen, Banken, Schulen, Universitäten etc. (vgl. z.B. Parker/Vaughan-Williams 2012). Bethan Loftus (2013) spricht beispielsweise von einer Vertiefung von Grenzen. Jedoch auch hierbei spielen Sprache und die Konstruktion neuer dominanter Diskursströmungen und Bedeutungsschemata eine tragende Rolle. Der Topos Sicherheit hat sich zu einem der zentralen diskursiven Elemente etabliert - auch aus einer historischen Sicht (vgl. Huysmans 2000; Bigo 2002). Durch eine historische Diskurs- und Policy-Analyse wurde nachgewiesen, wie Versicherheitlichung sukzessiv eine politische Legitimierung erhalten hat und dadurch politische Maßnahmen und ihre Auswirkungen normalisiert wurden (vgl. Vollmer 2014; 2017b).

Gleichzeitig haben jedoch migrantische Bewegungen die Prinzipien und Prozesse der Versicherheitlichung auf ihre Umsetzbarkeit geprüft und ihre Legitimation herausgefordert. Migrationsund Fluchtbewegungen über das Mittelmeer haben dazu geführt, dass humanitäre Fragestellungen vordergründiger wurden und beispielsweise vermehrt auf das Prinzip des Festungsbaus und des Rechtsentzugs durch bordering aufmerksam gemacht wurde (vgl. z.B. Ticktin 2011; 2014). Die globale humanitäre Krise, wie sie an europäischen und anderen Grenzen weltweit vorzufinden ist, hat ihre Wurzeln in der Konstruktion von Staaten und Grenzziehungen jeglicher Art, die imperialistische, rassistische und andere exkludierende Prozesse und Entwicklungen mit sich brachten bzw. nach sich zogen. Durch Globalisierungsprozesse, transnationale Praktiken und Migrationsbewegungen sowie zunehmende Diversität und wachsende Ungleichheit findet sich ein diffuses Verhältnis vom Innen und Außen und der konstitutiven Funktionen von Grenzen. Es treten nicht gesicherte, sondern zunehmend verunsicherte Wahrnehmungen

2 Gegenwärtig hat die Coronavirus-Krise 2020 allerdings gezeigt, dass Grenze nach wie vor insbesondere eine repressive Funktion hat und dass Staaten die Macht haben, Grenze durchzusetzen und nahezu jegliche Migration zu unterbinden. 
und Bedeutungen von dem Objekt Grenze auf (vgl. Vollmer 2019). Es deutet auf die Frage hin, ob sich die analytische Linse bezüglich des Konzepts und des Gegenstands Grenze ändern sollte? Innovative analytische Ansätze aus der Disziplin der Sicherheitsstudien (security studies), wie z.B. die Anwendung von endemischen Sicherheitskonzepten (vernacular security; vgl. Bubandt 2005; Vaughan-Williams/Stevens 2016), können wegweisend sein, um Fragen der Sicherheit aus der Perspektive der Bevölkerung sowie auch der Migrierenden anstatt aus der Perspektive des Staates oder der beteiligten politischen Akteure zu beantworten (vgl. Vollmer 2019).

Es stellen sich neue Fragestellungen bezüglich des Verhältnisses von Grenzen und Migration. Anzeichen für Xenophobie und ihre Implikationen haben sich seit 2014 in mehreren europäischen Ländern offenkundig gehäuft. Die Symbolik der Unsicherheit von Grenzen und die damit assoziierte schwindende Kontrolle des Inneren und des Äußeren, der suggerierten unkontrollierten Migration, hat neue politische Diskurse mobilisiert und neue politische Akteure gestärkt. Unsicherheit wurde durch populistische Strategien instrumentalisiert. Neue Feindbilder wurden produziert und neue Grenzen und Abgrenzungen sollen um ein weiteres Mal in der Geschichte für Recht, Ordnung und Sicherheit sorgen. Der Angst vor dem imaginären Feind - derzeit nochmals verstärkt durch die Coronavirus-Krise 2020 - soll mit dem Glauben an neue Grenzkonstruktionen Einhalt geboten werden. Trotz fortschreitender globaler Integration scheint sich im 21. Jahrhundert paradoxerweise eine Renaissance der Grenze abzuzeichnen.

\section{Fazit}

Zwischen Migration und Grenzen besteht eine dynamische Interdependenz. Sie werden von einem reziproken Verhältnis bestimmt, wobei gleichzeitig eine wachsende Pluralität an Bedeutungen von Migration und Grenzen beobachtet werden kann.

In der Grenzforschung stellen mikrosoziologische und ethnografische Ansätze wie auch Diskursforschung neue zukunftsträchtige Forschungszweige dar (siehe auch Lehner in diesem Band). Die migranten- und flüchtlingszentrierte Forschung wird zunehmend durch die Erforschung der street level bureaucrats an der Grenze ergänzt. Empirische (ethnografische oder quantitative) Erforschung tatsächlicher Abläufe an Grenzen, die der Politikproduktion sowie der Durchführung politischer Richtlinien behilflich sein kann, darf nicht vernachlässigt werden. Aus diesem Grund besteht ein Mangel, Auswirkungen der Politikproduktion sowie der nicht intendierten Nebeneffekte oder dem gänzlichen Versagen von politischen Maßnahmen zu erfassen. Insbesondere die Erforschung von Protesten an Grenzen und gegen Einreisebeschränkungen könnte eine Brücke zwischen diesen beiden Aufgaben bilden. Hierbei bleibt allerdings eine große Hürde bestehen - und das ist der Zugang zum Feld Grenze oder Grenzanlagen. Auch wenn der Zugang zum Feld zugegebenermaßen eine Herausforderung darstellt und mitunter unbequem sein mag, so bleibt die Erforschung von Grenzen oder Grenzanlagen doch eine zentrale Aufgabe der Forschung.

Ebenso versprechen innovative Methoden der Diskursforschung durch Anwendung von neuen Technologien und Verfahren in Zukunft die Möglichkeit, große Textkorpora in Verbindung mit Visualisierungen zu erforschen. Weitere Entwicklungen innovativer Mixed-methods-Ansätze nehmen beispielsweise eine weitere Triangulierung von Methoden der Diskursforschung mit der ethnografischen Feldforschung oder teilnehmenden Beobachtungen vor.

Zusätzlicher Motor der komplementären Phänomene wachsender Migrationsbewegungen und der Renaissance von Abgrenzungen ist das veränderte politische Klima. Politischer Populismus 
nutzt die steigende Diffusion der Phänomene sowie die sich ändernde politische Kommunikation: durch eine strategische Dämonisierung in Migrationsdiskursen, die von produktiven Narrativen ,neuer Helden', ,neuer Schurken' und ,neuen unschuldigen Opfern' gespeist, ausgeschmückt und effektiv mobilisiert werden (vgl. Anderson 2013), bis hin zur Verteufelung von Migration im Zuge der Coronavirus-Krise zeichnet sich keine Empörung in der Öffentlichkeit ab, sondern eine ernst zu nehmende subversive Aushöhlung liberaler Demokratien. Politische Kulturen leiden in Europa unter einem Neopopulismus, der vor allem in Feldern wie Migration in Verbindung mit Grenzen erste Erfolge verzeichnet. Eine ständige Ausweitung unerwünschter Kategorien von Außenseitern und Außenseiterinnen oder ,anderen` zeigt sich jedoch nicht lediglich in populistischen Bewegungen, sondern auch in den etablierten politischen Kreisen und Parteien (vgl. z.B. Koppetsch 2019). Diese Entwicklung steht für einen notwendigen Aufruf an die Grenz- und Migrationswissenschaft, innovative Perspektiven und Ansätze in ihre wissenschaftlichen Agenden nachhaltig aufzunehmen.

Im Rahmen dessen stellt sich die Frage nach der ausbleibenden öffentlichen Empörung in den Zielstaaten von Migration aufgrund von Aussagen von politischen Akteuren und Akteurinnen innerhalb Europas oder der Vereinigten Staaten. Scheinbar besteht ein legitimierter diskursiver Raum für Donald Trump, irreguläre Migranten und Migrantinnen als „Tiere“ zu bezeichnen (vgl. Hirschfeld Davis 2018). Das heißt, wenn diskursive Kräfte regionale oder nationale Migrationsregime formen (vgl. z.B. Wodak 2015; Rheindorf/Wodak 2020), muss sich Forschung damit dringend auseinandersetzen.

\section{Weiterführende Literatur}

Agier, Michel (2016): Borderlands. Cambridge: Polity Press.

Bauder, Harald (2017): Migration Borders Freedom. London: Routledge

Betts, Alexander (Hrsg.) (2011): Global migration governance. Oxford: Oxford University Press.

Crawley, Heaven/Düvell, Franck/Sigona, Nando/McMahon, Simon/Jones, Catherine (2017): Unravelling the Mediterranean Migration Crisis. Bristol: Policy Press.

Nail, Thomas (2016): Theory of Border. Oxford: Oxford University Press.

\section{Literaturverzeichnis}

Adamson, Fiona (2006): Crossing Borders: International Migration and National Security. In: International Security 31, H. 1, S. 165-199.

Anderson, Bridget (2013): Us and Them? The Dangerous Politics of Immigration Control. Oxford: Oxford University Press.

Anderson, Malcolm (1997): The political science of frontiers. In: Ganster, Paul/Sweedler, Alan/ Scott, James/ Eberwein, Wolf-Dieter (Hrsg.): Borders and border regions in Europe and North America. San Diego: San Diego State University Press, S. 27-45.

Appadurai, Arjun (2013): The Future as Cultural fact: Essays on the Global Condition. London: Verso.

Balibar, Etienne (2004): We the people of Europe? Reflections on transnational citizenship. Princeton: Princeton University Press.

Balibar, Etienne (2009): Europe as borderland. In: Environment and Planning D: Society and Space 27, H. 2, S. 190-215.

Basch, Linda/Glick-Schiller, Nina/Blanc, Christina (Hrsg.) (1994): Nations Unbound. London: Routledge.

Bauder, Harald (2017): Migration Borders Freedom. London: Routledge.

Bauman, Zygmund/Lyon, David (2012): Liquid Surveillance. Cambridge: Polity.

Bhagwati, Jagdish (2003): Borders beyond control. In: Foreign Affairs 82, H. 1, S. 98-104.

Betts, Alexander (Hrsg.) (2011): Global migration governance. Oxford: Oxford University Press.

Bigo, Didier (2002): Security and Immigration: Towards a Critique of the Governmentality of Unease. In: Alternatives 27, S. 63-92.

Bommes, Michael/Geddes, Andrew (2000): National welfare state, biography and migration. In: Bommes, Michael/Geddes, Andrew (Hrsg.). Immigration and welfare: challenging the borders of the welfare state. Abingdon: Routledge, S. 1-12. 
Broeders, Dennis (2007): The New Digital Borders of Europe: EU Databases and the Surveillance of Irregular Migrants. In: International Sociology 22, H. 1, S. 71-92.

Bubandt, Nils (2005) Vernacular Security: The Politics of Feeling Safe in Global, National, and Local Worlds. In: Security Dialogue 36, H. 3, S. 275-296.

Buzan, Barry (1991): People, States and Fear: An Agenda for International Security Studies in the PostCold War Era. 2. Aufl., London: Harvester Wheatsheaf.

Caplan, Jane/Torpey, John (Hrsg.) (2001): Documenting Individual Identity: The Development of State Practices in the Modern World. Princeton: Princeton University Press.

Carling, Jorgen (2002): Migration in the age of involuntary immobility: Theoretical reflections and Cape Verdean experiences. In: Journal of Ethnic and Migration Studies 28, H. 1, S. 5-42.

Castles, Stephen/Miller, Mark (1998): The Age of migration. Houndmills: Macmillan.

Collinson, Sarah (1993): Beyond borders: West European Migration Policy towards the 21st century. London: Royal Institute of International Affairs.

Collyer, Michael/deHaas, Hein/Düvell, Franck (2012): Critical Approaches to Transit Migration. In: Population, Space and Place 18, H. 4, S. 407-414.

Cooper, Anthony/Rumford, Chris (2013): Monumentalising the Border: Bordering Through Connectivity. In: Mobilities 8, H. 1, S. 107-124.

Côté-Boucher, Karine/Infantino, Federica/Salter, Mark (2014): Border security as practice: An agenda for research. In: Security Dialogue 45, H. 3, S. 195-208.

Crawley, Heaven/Düvell, Franck/Sigona, Nando/McMahon, Simon/Jones, Catherine (2017): Unravelling the Mediterranean Migration Crisis. Bristol: Policy Press.

Cuttitta, Paolo (2014): „Borderizing“ the Island: Setting and Narratives of the Lampedusa „Border Play“. In: ACME: An International E-Journal for Critical Geographies 13, H. 2, S. 196-219.

Düvell, Franck (2006): Illegal migration. Houndmills: Palgrave Macmillan.

Düvell, Franck (2017): „Voice“ or „exit“? From the streets of Syria to the borders of the EU. In: Geografie 122, H. 4, S. 429-448.

Düvell, Franck/Molodikova, Irina/Collyer, Michael (2014): Transit Migration in Europe. Amsterdam: Amsterdam University Press

Eschbach, Karl/Hagan, Jacquelin/Rodriguez, Nestor/Hernández-León, Ruben/Bailey, Stanley (1999): Death at the Border. In: International Migration Review 33, H. 2, S. 430-454.

Europäische Kommission (2019): 2019 Annual Report on Intra-EU Labour Mobility. Brüssel: Europäische Kommission.

Faist, Thomas (2016): Cross-Border Migration and Social Inequalities. In: Annual Review of Sociology 42 , H. 1, S. 323-346.

Fassin, Didier (2012): Humanitarian reason: A moral history of the present. Berkeley and London: University of California Press.

Gallup (2017): Number of Potential Migrants Worldwide Tops 700 Million. www.news.gallup.com/poll/2 11883/number-potential-migrants-worldwide-tops-700-million.aspx, 16.8.2018.

Gammeltoft-Hansen, Thomas (2011): The Externalisation of European Migration Control and the Reach of International Refugee Law. In: Guild, Elspeth/Minderhoud, Paul (Hrsg.): The First Decade of EU Migration and Asylum Law, Anniversary volume of the European Journal of Migration and Law. Leiden: Brill, S. 273-298.

Genova, Nicholas De (2002): Migrant „Illegality“ and Deportability in Everyday Life. In: Annual Review of Anthropology 31, H. 1, S. 419-447.

Goldin, Ian (2018): Migration and the Economy: Economic Realities, Social Impacts and Political Choices. Oxford: University of Oxford.

Harris, Nigel (2007): The economics and politics of the free movement of people. In: Pécoud, Antoin/Guchteneire, Paul de (Hrsg.): Migration without borders. Oxford: Berghahn. S. 33-50.

Hayter, Theresa (2000): Open borders, The case against immigration controls. London: Pluto.

Heather, Peter (2010): Empires and Barbarians: The Fall of Rome and the Birth of Europe. Oxford: Oxford University Press.

Hess, Sabine/Tsianos, Vassilis (2010): Ethnographische Grenzregimeanalyse. Eine Methodologie der Autonomie der Migration. In: Hess, Sabine/Kasparek, Bernd (Hrsg.): Grenzregime: Diskurse, Praktiken, Institutionen in Europa. Berlin: Assoziation A, S. 243-264.

Hirschfeld Davis, Julie (2018): Trump Calls Some Unauthorized Immigrants „Animals“ in Rant. In: New York Times, 16.5.2018.

Hoerder, Dirk (2002): Cultures in Contact. Durham: Duke University Press.

Houtum, Henk van/Naerssen, Ton van (2001): Bordering, ordering and othering. In: Tijdshrift voor Economische en Sociale Geografie 93, H. 2, S. 125-136. 
Huysmans, Jef (2000): The EU and the Securitization of Migration. In: Journal of Common Market Studies 38, H. 5, S. 751-777.

Huysmans, Jef (2006): The politics of insecurity: Fear. Migration and asylum in the EU. London and New York: Routledge.

Infantino, Federica (2016): Outsourcing border controls. New York: Palgrave Macmillan.

International Labour Office (ILO) (2015): ILO Global Estimates of Migrant Workers and Migrant Domestic Workers: Results and Methodology. Genf: ILO.

Khosravi, Shahram (2010): „Illegal“ traveler. An auto-ethnography of borders. Houndmills: Palgrave Macmillan.

Koppetsch, Cornelia (2019): Die Gesellschaft des Zorns. Rechtspopulismus im globalen Zeitalter. Bielefeld: transcript.

Krasner, Stephen (1982): Structural Causes and Regime Consequences: Regimes as Intervening Variables. In: International Organization 36, H. 2, S. 85-205.

Last, Tamara/Mirto, Giorgia/Ulusoy, Orçun/Urquijo, Ignacio/Harte, Joke/Bami, Nefeli/Pérez Pérez, Marta/Macias Delgado, Flor/Tapella, Amélie/Michalaki, Alexandra/Michalitsi, Eirini/Latsoudi, Efi/Tselepi, Naya/Chatziprokopiou, Marios/Spijkerboer, Thomas (2017): Deaths at the borders database: evidence of deceased migrants' bodies found along the southern external borders of the European Union. In: Journal of Ethnic and Migration Studies 43, H. 5, S. 693-712.

Lehner, Sabine/Rheindorf, Markus (2018): „Fortress Europe“: Representation and argumentation in Austrian media and EU press releases on border policies. In: Dell`Orto, Giovanna/Wetzstein, Irmgard (Hrsg.): Refugee News, Refugee Politics: Journalism, Public Opinion and Policymaking in Europe. London: Routledge, S. 40-55.

Loftus, Bethan (2013): Border regimes and the sociology of policing. In: Policing and Society 25, H. 1, S. $115-125$.

Lutterbeck, Derek (2006): Policing Migration in the Mediterranean. In: Mediterranean Politics 11, H. 1, S. 59-82.

Mezzadra, Sandro (2010): Autonomie der Migration - Kritik und Ausblick, In: Grundrisse 34, S. $22-29$.

Moulier-Boutang, Yann (2002): Thesen zur Autonomie der Migration und zum notwendigen Ende des Regimes der Arbeitsmigration. In: Jungle World 15, 3.4.2002. www.jungle-world.com/artikel/2002/14/2 4171.html, 23.3.2003.

Mountz, Alison (2010): Seeking Asylum. Human smuggling and bureaucracy at the border. Minneapolis: Minnesota University Press.

Paasi, Anssi/Prokkola, Eeva-Kaisa (2008): Territorial Dynamics, Cross-border Work and Everyday Life in the Finnish-Swedish Border Area. In: Space and Polity 12, H. 1, S. 13-29.

Papadopoulos, Dimitris/Tsianos, Vassilis (2013): After citizenship: autonomy of migration, organisational ontology and mobile commons. In: Citizenship Studies 17, H. 2, S. 178-196.

Parker, Noel/Vaughan-Williams, Nick (2012): Critical Border Studies: Broadening and Deepening the „Lines in the Sand“ Agenda. In: Geopolitics 17, H. 4, S. 727-733.

Pecoud, Antoine/Guchtenaire, Paul de (2006): International migration, border controls and human rights: assessing the relevance of a right to mobility. In: Journal of Borderland Studies 21, H. 1, S. 69-86.

Petryna, Adriana/Follis, Karolina (2015): Risks of Citizenship and Fault Lines of Survival. In: Annual Review of Anthropology 44, H. 1, S. 401-441.

Raghuram, Parvati/Piper, Nicola (2011): Women and migration in Asia - eroding borders, new fixities. In: Wastl-Walter, Doris (Hrsg.): The Ashgate Research Companion to Border Studies. Farnham: Ashgate, S. 529-548.

Rheindorf, Markus/Wodak, Ruth (2020): Building „Fortress Europe“: Legitimizing Exclusion from Basic Human Rights. In Rheindorf, Markus/Wodak, Ruth (Hrsg.): Sociolinguistic Perspectives on Migration Control: Language Policy, Identity and Belonging. Bristol: Multilingual Matters, S. 116-147.

Rodriguez, Nestor (1996): The Battle for the Border: Notes on Autonomous Migration, Transnational Communities, and the State. Social Justice 23, H. 3, S. 21-37.

Rumford, Chris (2006): Theorizing borders. European Journal of Social Theory 9, H. 2, S. 155-169.

Ryan, Bernard/Mitsilegas, Valsamis (Hrsg.) (2010): Extraterritorial immigration control. Leiden: Martinus Nijhoff.

Scheel, Stephan (2013): Autonomy of Migration Despite Its Securitisation? Facing the Terms and Conditions of Biometric Rebordering. In: Millennium - Journal of International Studies 41, H. 3, S. 575-600.

Shields, Rob (2006): Boundary-thinking in theories of the present: The virtuality of reflexive modernization. In: European Journal of Social Theory 9, H. 2, S. 223-237.

Schindel, Estela (2016): Bare life at the European borders. Entanglements of technology, society and nature, In: Journal of Borderlands Studies 31, H. 2, S. 219-234. 
Shrestha, Nanda R. (1987): International policies and migration behaviour: a selective review. In: World Development 15, H. 3, S. 329-345.

Takikawa, Hirohide (2016): Can we justify the welfare state in an age of globalisation? Toward complex borders, In: Archiv für Rechts- und Sozialphilosophie 92, H. 1, S. 15-27.

Ticktin, Miriam (2011): Casualties of care. Immigration and the politics of humanitarianism in France. Berkeley: University of California Press.

Ticktin, Miriam (2014): Transnational Humanitarianism. In: Annual Review of Anthropology 43, S. 273 289.

Triandafyllidou, Anna/Maroukis, Thanos (2012): Migrant smuggling. Houndmills: Palgrave Macmillan.

Tsianos, Vassilis/Hess, Sabine (2010): Ethnographische Grenzregimeanalyse. In: Hess, Sabine/Kasparek, Bernd (Hrsg.): Grenzregime. Berlin: Assoziation A, S. 243-264.

Tuckett, Anna (2018): Rules, Paper, Status: Migrants and Precarious Bureaucracy in Contemporary Italy. Stanford: Stanford University Press.

United for Intercultural Action (2018): UNITED Updated List of 34,361 Refugee Deaths. www.unitedagai nstracism.org/blog/2018/06/20/press-release-united-list-of-34361-refugee-deaths-published-in-the-guardi an/, 14.10.2019.

United Nations (UN) (1997): Concise report on world population monitoring, 1997: international migration and development, Commission on Population and Development. www.un.org/documents/ecosoc/cn 9/1997/ecn91997-2.htm, 19.8.2010.

United Nations (UN) (2013): International Migration Policies: Government Views and Priorities. www.un. org/en/development/desa/population/publications/pdf/policy/InternationalMigrationPolicies2013/Report \%20PDFs/z_International\%20Migration\%20Policies\%20Full\%20Report.pdf, 22.5.2014.

United Nations Department of Economic and Social Affairs Statistics Division (UNDESA) (1998): Recommendations on Statistics of International Migration. In: Statistical Papers Series M 58, Rev. 1. New York: United Nations.

United Nations Department for Economics and Social Affairs (UNDESA) (2017): International migration report 2017. www.un.org/development/desa/publications/international-migration-report-2017.html, 11.11.2018.

United Nations High Commissioner for Refugees (UNHCR) (1997): The state of the world's refugees. www.unhcr.org/3eb7bb534.html, 5.5.2005.

United Nations High Commissioner for Refugees (UNHCR) (2018): Figures at a glance. www.unhcr.org/fi gures-at-a-glance.html, 4.7.2019.

United Nations High Commissioner for Refugees (UNHCR) (2020): What is a refugee?. www.unrefugees. org/refugee-facts/what-is-a-refugee/, 1.4.2020.

United Nations World Tourism Organization (UNWTO) (2016): A decade of world tourism. www.cf.cdn. unwto.org/sites/all/files/pdf/unwto_guilin_2016_jk_fin_1.pdf, 16.8.2017.

United Nations World Tourism Organization (UNWTO) (2018): World tourism barometer. www.media.u nwto.org/press-release/2018-01-15/2017-international-tourism-results-highest-seven-years, 5.8.2019.

Vaughan-Williams, Nick (2009): Border politics: The limits of sovereign power. Edinburgh: Edinburgh University Press.

Vaughan-Williams, Nick/Stevens, Daniel (2016): Vernacular Theories of Everyday (In)security: The Disruptive Potential of Nonelite Knowledge. In: Security Dialogue 47, H. 1, S. 40-58.

Velde, Martin van der/Naerssen, Ton van (Hrsg.) (2015): Mobility and migration choices. Thresholds to crossing borders. London: Ashgate.

Vogel, Dita (2003): Migration Policy. In: Hansen, Randall/Gibney, Mathew (Hrsg.): Immigration and Asylum: From 1900 to the Present. Santa Barbara: ABC Clio, S. 421-426.

Vollmer, Bastian (2012): Making Light of Borders - The Case of the External EU Border. In: Migration Letters 9, H. 2, S. 131-140.

Vollmer, Bastian (2014): Policy Discourses on Irregular Migration in Germany and the United Kingdom. New York/Basingstoke: Palgrave Macmillan.

Vollmer, Bastian (2016): Ukrainian Migration and the European Union - dynamics, subjectivity, and politics. New York/Basingstoke: Palgrave Macmillan.

Vollmer, Bastian (2017a): Security or Insecurity? Representations of the UK Border in Public and Policy Discourses. In: Mobilities 12, H. 3, S. 295-310.

Vollmer, Bastian (2017b): The Continuing Shame of Europe: Discourses on migration policy in Germany and the UK. In: Migration Studies 5, H. 1, S. 49-64.

Vollmer, Bastian (2019): The paradox of border security - an example from the UK. In: Political Geography 71, H. 1, S. 1-19.

Waldron, Arthur (1990): The Great Wall of China: From History to Myth. Cambridge: Cambridge University Press. 
Walker, Rob (1993): Inside/Outside: International Relations as Political Theory. Cambridge: Cambridge University Press.

Walters, William (2002): Mapping Schengenland: denaturalizing the border. In: Environment and Panning D: Society and Space 20, H. 5, S. 561-580.

Walters, William (2009): Europe's borders. In: Rumford, Chris (Hrsg.): Sage Handbook of European Studies. London: Sage, S. 485-505.

Weber, Leanne/Pickering, Sharon (2011): Globalisation and borders. Death at the global frontier. Houndsmill: Palgrave Macmillan.

Wodak, Ruth (2015): The politics of fear: What right-wing populist discourses mean. London: Sage.

Yaghmaian, Behzad (2005): Embracing the Infidel. New York: Delacorte. 